\title{
Introducing Gamification in eHealth Platforms for Promoting Wellbeing
}

\author{
Antonios PARDOS ${ }^{\mathrm{a}, \mathrm{b}}$, Andreas MENYCHTAS ${ }^{\mathrm{a}, \mathrm{b}}$ and Ilias MAGLOGIANNIS ${ }^{\mathrm{b}, 1}$ \\ ${ }^{a}$ Bioassist $S . A$. \\ ${ }^{\mathrm{b}}$ Department of Digital Systems, University of Piraeus
}

\begin{abstract}
Gamification techniques are adopted by IT systems and applications in order to facilitate their adoption and motivate users to take advantage of specific application features. The current work presents a modern approach for the effective implementation of gamification features in a prototype eHealth application which encourages the daily use of the application, endorses the users to continuously monitor their health and promotes a healthier lifestyle. The implementation of this approach is modular and flexible in order to be easily applied in any similar system and tailor the provided features for user activity monitoring, analysis, feedback, and interactivity, to the specific requirements of the different usage scenarios.
\end{abstract}

Keywords. Gamification, eHealth, mHealth, IT systems

\section{Introduction}

eHealth solutions are considered as a type of intervention that can reach large numbers of people however, the low usage of the system from the participants is a recognized barrier to their sustainability and long-term success [1]. Romeo et. al. [2] mentioned that apps used to monitor physical activity are most effective in the short term, and the engagement declines over time which in turn limits the intervention effectiveness. The "use of game mechanics in non-gaming contexts" [3] is the technique known as gamification and is adopted for a variety of purposes in different contexts. The advantages of adopting those strategies might vary depending on the users' characteristics [4] or the scope of the application [5], and are related to both the user experience and how the users tend to engage with the app.

The most common and, historically, the traditional form of a gamification approach [6] is the Points - Badges - Leaderboards (PBL) triptych. More specifically, the user is awarded with numerical rewards (points) each time he completes a specific action within the application. Different users are ranked in descending order based on the points they have accumulated creating "Leaderboards", a concept borrowed from gaming design. A widely used approach is the "badges", which are given to users when they complete specific achievements. These achievements also demonstrate the level of familiarity of the user with the application and the change of his behavior to meet its purposes [6]. In this work we present an implementation of the badges approach, in the context of an eHealth application, as a fully flexible and modular IT solution which can be applied and

\footnotetext{
${ }^{1}$ Corresponding Author, Ilias Maglogiannis, Department of Digital Systems, University of Piraeus, 80, M. Karaoli \& A. Dimitriou St., 18534 Piraeus, Greece; E-mail: imaglo@unipi.gr.
} 
integrated in any similar system. The combination of eHealth systems with gamifications features has the advantage of motivating the users to change their daily behaviors in a healthier way. Physical activity boost is a major outcome that the users of eHealth applications can achieve through gamification, and in the proposed proof-of-concept scenario it is also combined with the continuous measurement of other biosignals and the daily use of the application. Technically, the approach focusses on two aspects, an advanced model for efficient data management and analysis, and also the multimodal mechanisms required to calculate the badges levels. Both are considered of major importance for the efficient and effortless integration into any eHealth system.

\section{Implementation}

The implementation of the approach has two main elements: a) the modeling of the gamification aspects in order to be interoperable and easily manipulated from the various components, and b) the use of the proper calculation techniques so that it can be easily integrated with existing eHealth solutions, which requires the implementation of specific functions as software modules. Core part of the badges model is the composite objects containing the number of levels for the various badges and their definition.

Each badge has a category depending on the activity that the user has to achieve. The proposed model of the badges' model includes the following four activities:

- Daily usage: The users have to use the app (or specific app features) daily in order to upgrade his badge levels.

- Measurements: The measurements are processed in different timeframes and aggregation functions (e.g. sum, average, max, min) are applied to calculate the level.

- $\quad$ Sleep: The users have to achieve a certain sleep score every night (e.g. duration, quality) to be promoted to the next level.

- $\quad$ Steps: The users have to make a number of steps every day and stay active.

The system is able to calculate different aggregation metrics which are related with the specific activities of each user. This approach gives the system operators the option to define any number of badges, in any category, referencing in the model the function, or the combination of functions which can be used to calculate the metrics of the user activity. Aggregation functions such as min, max, sum, count on raw data can be applied in different time periods for each activity in order to easily perform the calculation of the levels for complex badge types. An example on this approach is to set badges for periods $\mathrm{P}$ in $\{$ Daily, Weekly, Monthly $\}$, with aggregation function $f u n c=s u m$, and data $=$ STEPS or data $=S L E E P$. The data are then summarized for the requested periods (e.g. one of Daily, Weekly, Monthly), the calculation functions will produce the intermediate scores and finally the badge levels. The users' Personal Health Records (PHRs), or other system data like the time the user accessed the app, are the raw input for the calculations and are produced by querying the data storage of the eHealth system. The raw data are transformed into objects that only hold the amount of information required for calculations. It should be noted that the proposed model, also supports caching of previous calculations outcomes allowing for incremental implementation of calculation operations. This means that for long periods, the data are continuously calculated, as they arrive, and if there are no changes on the level, they are cached in order to avoid querying 
again the data from the database and aggregating the different periods from the beginning. The general approach for the calculation of the users' progress in such badges is illustrated in Figure 1.

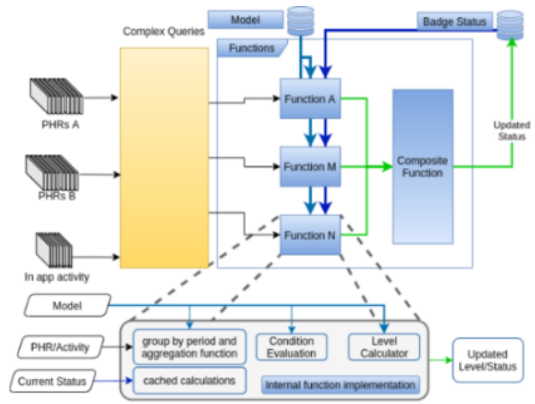

Figure 1. Model-driven composition of functions for the calculation of level status

\section{System in practice}

The approach has been validated in the context of eHealth applications of BioAssist SA [9]. The model and the calculation functions have been implemented as cloud functions according to the design concept of microservices that is followed by the particular platform. The operation of the solution demonstrates a fully flexible and modular design which can be applied and integrated in any similar system. The badge models for this prototype system are focused on the interaction with users and are tailored to the need of remote patient monitoring as presented in the Figure 2.

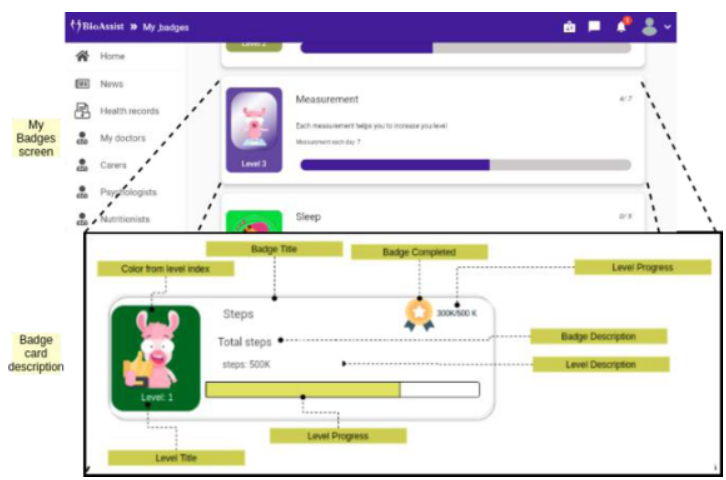

Figure 2. The "My Badges" screen and a sample badge card

The user interface was designed in order to address the main objective to provide feedback on the user's progress and educate them how to accomplish the various health and wellbeing related goals. Interviews with users and professionals, and analysis of their feedback was fundamental for the improvement of the overall design and of the experience. All badges have a number of levels to ensure the long-term gamification feature within the application. Every badge has an icon and the levels in the same badge are distinguished by the background color of the icon. The model also supports different icons and messages for each level. On technical and operation level, each model is stored in a key-value database as a json format and contains all the badges and the levels that each badge has. Twice a day and also after specific user interactions with the app, the 
system executes the function that is defined in the model which calculates the scores and updates the status of the badges. The results are stored in the database and the users are notified in-app and via email for their progress.

\section{Conclusions and Future Work}

The role of gamification as a feature in eHealth applications seems to have positive effects on the motivation of users in order to achieve their targets, increase their physical activity, and adhere to a healthcare plan. In this work we presented an implementation of a gamification approach based on "badges", in the context of an eHealth application, as a fully flexible and modular IT solution which can be applied and integrated in any similar system. The extensive evaluation of the approach is ongoing in the context of pilot activities of the application which are currently operational. The proposed gamification strategy is expected to promote the use of the application, motive the users to exploit specific features of the eHealth system and most importantly help them improve their health and wellbeing. Extensions are already designed and will be presented in a future work. The extended approach will be focused on the implementation of leaderboards and user competitions which are expected to further enhance the user experience and provide more incentives for system use.

\section{Acknowledgements}

This research has been co-financed by the European Union and Greek national funds through the Operational Program Competitiveness, Entrepreneurship and Innovation, under the call RESEARCH - CREATE - INNOVATE (project code: MediLudus Personalised home care based on game and gamify elements T 2EK $\Delta$ K-03049).

\section{References}

[1] Hutchesson MJ, et al. eHealth interventions targeting nutrition, physical activity, sedentary behavior, or obesity in adults: A scoping review of systematic reviews. Obesity Reviews. 2021 Jun 23.

[2] Romeo A, et al. Can smartphone apps increase physical activity? Systematic review and meta-analysis. Journal of medical Internet research. 2019;21(3):e12053.

[3] Deterding S, Dixon D, Khaled R, Nacke L. From game design elements to gamefulness: defining" gamification". InProceedings of the 15th international academic MindTrek conference: Envisioning future media environments 2011 Sep 28; p. 9-15.

[4] Koivisto J, Hamari J. Demographic differences in perceived benefits from gamification. Computers in Human Behavior. 2014 Jun 1;35:179-88.

[5] Rajanen M, Rajanen D. Usability benefits in gamification. In GamiFIN. 2017;87-95.

[6] Hamari J, Koivisto J, Sarsa H. Does gamification work?--a literature review of empirical studies on gamification. In 2014 47th Hawaii international conference on system sciences, IEEE. 2014, January;3025-3034).

[7] Costa R, et al. SmartLife smart clothing gamification to promote energy-related behaviours among adolescents. In 2017 International Conference on Engineering, Technology and Innovation (ICE/ITMC), IEEE.2017 Jun 27;1489-1495).

[8] Tabak M, De Vette F, Van DIjk H, Vollenbroek-Hutten M. A game-based, physical activity coaching application for older adults: Design approach and user experience in daily life. Games for health journal. 2020 Jun 1;9(3):215-26.

[9] BioAssist | Innovative eHealth solutions. (2021). Retrieved 9 September 2021. Available from: https://bioassist.eu/. 